advancement. Mr. Brown will find that the real test of the National Plan will lie in the way in which it channels the national effort, and not the least the way in which it affects the civil research and devclopment effort.

\section{PANGLOSS OR PLOTINUS}

The Uniqueness of Biological Materials

By A. E. Needham. (International Series of Monographs in Pure and Applied Biology. Division: Zoology, Vol. 25.) Pp. xix +593 . (London and Now York: Pergamon Press, 1965.) 100s. net.

$\triangle$ FEW years ago those who followed in the footsteps of L. J. Honderson and argued that the properties of elements and compounds were adapted, to an extent that was unlikely to be accidental, to the requirements of life and that therefore our form of lifo was likely to be the only one, had little reuson to fear refutation. It takes rather more self-confidence to argue this case now, for space travel could produco contradictory evidence. Those who maintain that Henderson paid insufficient attention to the probability that, in evolving here in the way it did, life exploited the existing situation opportunistically, and could be different in a different environment, are still on reasonably safe ground; they can 'have it both ways'. If organisms should bo found on another planet that depend on a basic biochemistry similar to that used on Earth, it may mean no more than that both have come (or just conceivably havo been sent) from a common source. If an alien form of life should be found, our cuse will be proved. The two (or more) systems might be called chthonobiology and xenobiology, and the investigation of the extent to which each is adapted to local conditions will make a fascinating study.

While waiting for the possible start of this extreme form of comparativo biochemistry, it is as well to survey the extent to which organisms have adapted themselves to local conditions and exploited all chemical potentialities. Needham attempts this comprehensibly and, on the whole, successfully. The Uniqueness of Biological Materials is not without faults; there is a grout deal of matter in it with no obvious relevance to the theme, there are several factual errors, and the citing of references is capricious. Too many references ars to secondary or even popular sourees, and oflen relate to matter that is dealt with in text-books, whereas interesting, and sometimes improbable, statements slip in with no authority. Nevertheless, it is a serious and original attempt to deal with an important subject and ropresents the fruit of a vast amount of reading.

Threc-quarters of tho book is devoted to carbon compounds. Those aspects of biochemistry that are nearly universal are outlined and some of the more intriguing by-ways are mentioned. Needham not only discusses the molecules that are used but also those that are not--or that aro used rarely. This is fundamental to the proposition implied by his title. The frequent reappearance of the same molecules could arise because these are the only ones able to bring about the required reactions effectively; because the dependence of organisms on one another in food chains forces the later links in the chain to comply with the pattern defined by the first member; or because, once a molecule is established in a well-integrated enzyme system, natural selection will tend to eliminate a variant, even if it uses a potentially better molecule, during the phase in which enzymes adapted to the now molecule are evolving. These points can be explored experimentally. Thus the diversity of phosphagens used in different phyla shows that different molecules can perform a similar function, but it is still by no means clear what advantage, if any, each has in the phylum in which it occurs.

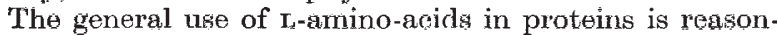
ably satisfactorily explained by invoking food chains and the greater instability of polymers containing both isomers compared to those containing only one. It is more difficult to explain the preference organisms show for the same twenty amino-acids. It would be interesting to know whether polymers containing some of the excluded amino-acids, for example, $L-\alpha$-amino-butyric acid, have any anomalous properties in vitro that could justify the exclusion. It is well known that an arnino-acid substitution, azetidine carboxylic acid for proline, or valine for glutamic acid, gives rise to a significantly different protein -but still a protein. Before we regard the usual aminoacids as unique we should know whether a polymer containing a significant proportion of the excluded amino-acids would be a protein at all.

The elements other than earbon are, naturally, considered in less detail. The manner in which the properties of such elements as iron and calcium adapt them for their observed biological roles is outlined. Those are common elements, so their genoral use is comprehensible; the rare use of such clements as barium, strontium and vanadium is more difficult to understand. Is this rarity simply a consequence of their patchy distribution on Earth's surface; or are these elements, in spite of the antiquity of the species that use them, intrinsically less suitable? This book gives no answer but, by presenting the evidence, it usefully calls attention to the existence of the problem. In the course of this discussion the conclusion is reached that biological processes do not alter the properties of the elements. That will be generally agreed, but it is surprising to find the issue discussed at all; one had thought the possibility was mooted only by those who think that the fuoride ion has different properties when coming directly from underground rather than from a bag, or that 'fixed' nitrogen differs in some way from that in dung.

The biochemical aspects of biology depend, in essence, on the interplay between morphology and enzyme action. If wo bear in mind the observed rate of dimensional change (4 per cent or more per million years), the plasticity and flexibility of proteins (excmplified by the ability of antibodies to conform to an immense range of antigens), and the 2,000 million years that have apparently been available for evolution, the observed congruence between chemical potentialities and the needs of organisms seems to raise no difficulties. The number of possible proteins is so vast that it is reasonable to expect them to cope with any chemical reactions that have possible 'survival value'. On this view our present stato may not yet be "the best in the best of all possible worlds", but we aro heading there. The rival view is that the congruence is improbably good; it leads to the concept of design, or to Plotinus's thesis that organisms and the basic properties of the environment act reciprocally on one another. The observa. tions made so far do not scem to force on us so radical an overthrow of accepted scientific canons. N. W. PIRIE

\section{LUNG CIRCULATION}

\section{The Lung Circulation}

By Prof. Dorningo M. Aviado. Vol. 1: Physiology and Pharmacology. Pp. xxiv $+1-590$. Vol. 2: Pathologic Physiology and Therapy of Diseases. Pp. xix + 591-1405. (London and New York: Pergamon Pross, 1965.) $300 s$. net, per sot.

T NTIL recent year's the pulmonary circulation was not a popular subject of investigation among either physiologists or clinicians. This was due, in part at least, to a lack of interest, and the belief, generally fostered by text-books of physiology, that the pulmonary circulation is effectively independent of the nervous system or of any humoral control. It had been repeatedly shown from tho late nineteenth century onwards that pulmonary vasomotor responses could be excited by both nervous and hormonal stimuli, but such evidence was generally 